\title{
Vaginal Metastasis of Urothelial Carcinoma Found Incidentally during Transurethral Resection of a Bladder Tumor
}

\author{
Kenji Ohgaki ${ }^{1,2}$, Kazutaka Horiuchi ${ }^{1,2}$, Fumiatsu Oka ${ }^{1,2}$, \\ Mitsuhiro Sato ${ }^{1,2}$ and Taiji Nishimura ${ }^{1}$ \\ ${ }^{1}$ Department of Urologic Surgery, Graduate School of Medicine, Nippon Medical School \\ ${ }^{2}$ Department of Urology, Nippon Medical School Musashi Kosugi Hospital
}

\begin{abstract}
A 71-year-old woman visited our hospital for routine follow-up cystoscopy and urine cytologic examination. During the preceding 3 years she had undergone left nephroureterectomy for a tumor of the left renal pelvis and had undergone transurethral resection three times for tumors of the urinary bladder and urethra. A small flare region on the posterior wall of the bladder was found with regular cystoscopy, and urine cytologic examination was positive for malignant cells. Computed tomography showed no distant metastasis and no right upper urinary tract mass. Transurethral resection was performed for the small flare region in May 2006. A papillary tumor of the vaginal wall was found incidentally during transurethral resection, and, therefore, transvaginal resection of the tumor was performed at the same time. Histologic examination of the excised genital lesion showed a G3 pT2 urothelial carcinoma, and the patient has been under observation since completion of external radiotherapy for the vaginal metastatic nodule.
\end{abstract}

(J Nippon Med Sch 2008; 75: 312-315)

Key words: vaginal metastasis, urothelial carcinoma, transurethral resection of bladder tumor

\section{Introduction}

Vaginal urothelial carcinoma following radical cystectomy has been reported, but vaginal metastasis of urothelial carcinoma without prior open surgery is extremely rare $^{1}$. Herein we report a case of urothelial carcinoma with vaginal metastasis after treatment of cancers of the left renal pelvis and the urinary bladder.

\section{Case Report}

A 71-year-old woman with gross hematuria and lumbar pain visited our hospital in April 2003. A tumor of the left renal pelvis was diagnosed with intravenous pyelography and computed tomography (CT), and left nephroureterectomy was performed in May 2003. Histological examination confirmed a G2 pT3 transitional cell carcinoma (TCC) of the left renal pelvis. The patient underwent transurethral resection three times from August 2003 through

Correspondence to Kenji Ohgaki, MD, PhD, Department of Urology, Nippon Medical School Musashi Kosugi Hospital, 1-396 Kosugi-cho, Nakahara-ku, Kawasaki, Kanagawa 211-8533, Japan

E-mail:kogaki@nms.ac.jp

Journal Website (http://www.nms.ac.jp/jnms/) 

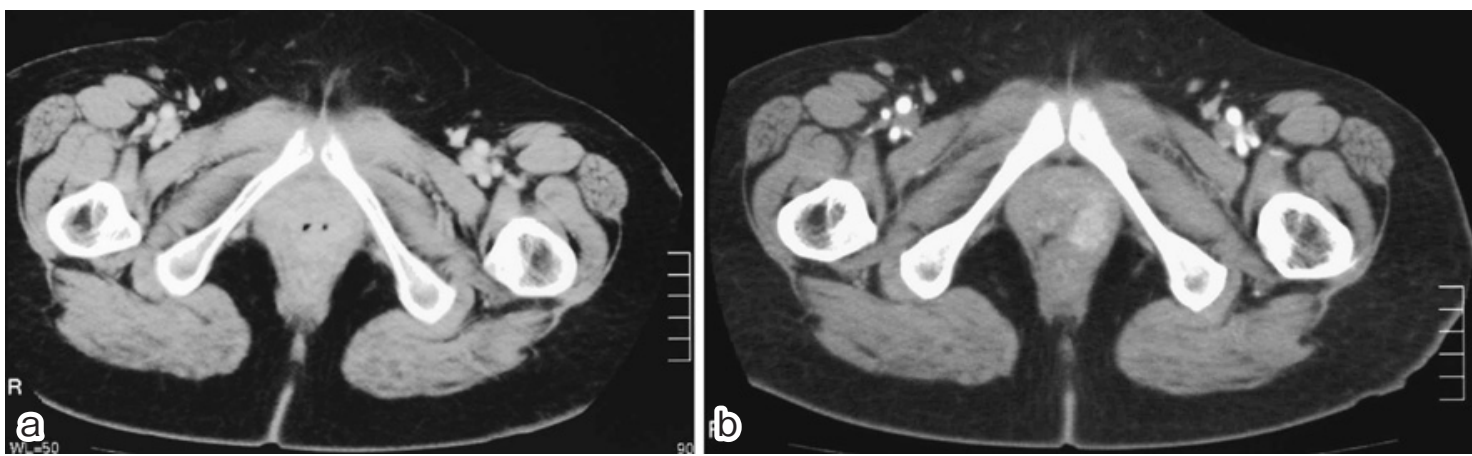

Fig. 1

a: Contrast-enhanced $\mathrm{CT}$ scan showing almost normal vagina

b: Contrast-enhanced CT scan showing a mass in the left side of the vagina

December 2004 with regular cystoscopy because of multiple papillary tumors of the bladder or urethra. The histological examination of each tumor confirmed G1-2 pTa-T1 TCC of the bladder, and histologic examination in August 2003 also confirmed a G2 pT1 TCC of the urethra. The patient subsequently received 6 cycles of intravesical instillation of Bacille Calmette-Guérin vaccine from August 2003 through March 2004.

The patient again complained of gross hematuria in January 2006. Urine cytologic examination was positive for malignant cells. The findings of cystoscopy, intravenous pyelography, and CT (Fig. 1a) were almost normal, but a flare region was observed on the posterior wall of the bladder. A tumor was not found in the bladder, but transurethral resection of the flare region was performed in May 2006. A papillary tumor on the vaginal wall was revealed when the endoscope accidentally reflected the vagina during transurethral resection, and transvaginal resection was performed for the tumor on the vaginal wall. Histological examination confirmed a G3 pT2 urothelial carcinoma of the vagina (Fig. 2) and chronic cystitis. The residual nodule $(25 \times 13 \mathrm{~mm})$ on the posterior wall of the vagina was examined with magnetic resonance imaging and CT (Fig. 1b) for staging. We obtained informed consent for radical surgery but could not obtain consent for urinary diversion. We then introduced the patient to another hospital because she hoped to obtain a second opinion. She was subsequently treated with external irradiation of the vaginal metastatic nodule and left

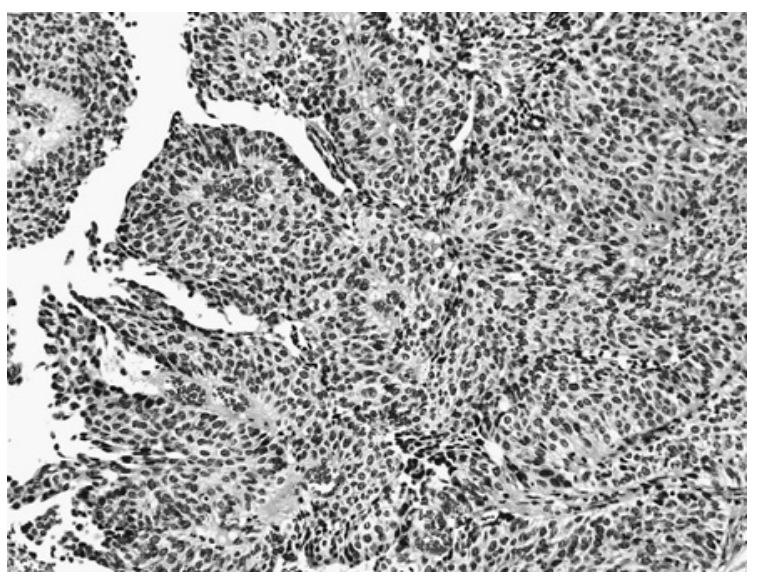

Fig. 2 G3 papillary urothelial carcinoma with invasion of the vagina

inguinal adenopathy (a total of 60 Gy in each case) and is now under observation.

\section{Discussion}

Metastasis of urothelial cancer to the vagina is extremely rare, with only 15 cases, including the present case, reported worldwide. The average interval from the diagnosis of urothelial cancer to vaginal metastasis in these cases was 4.3 years (range: 0-14 years), and the treatment for the vaginal metastatic nest was endoscopic resection in 11 cases $^{2-5}$ and total hysterovaginectomy in 2 cases $^{6.7}$. A breakdown of the cases of vaginal metastasis of urothelial carcinoma is shown in Table 1. Noordzij et al. ${ }^{8}$ have proposed that implantation of shed tumor cells in tissues "downstream" during micturition or resection is the only plausible mechanism of metastasis. Some authors ${ }^{24.68}$ have 
K. Ohgaki, et al

Table 1 The Cases of Vaginal Metastasis of Urothelial Carcinoma

\begin{tabular}{|c|c|c|c|c|c|c|}
\hline \multirow{2}{*}{ author } & \multirow{2}{*}{ year } & \multicolumn{2}{|r|}{ vaginal recurrence } & \multicolumn{3}{|c|}{ past history } \\
\hline & & age & pathological diagnosis & age & organ & pathological diagnosis \\
\hline Murai et al. & 1989 & 60 & invasive, papillary & 60 & lt. renal pelvis & invasive poorly diff. \\
\hline Fetissof et al. & 1990 & 76 & non-invasive, G1 & 71 & rt. renal pelvis & non-invasive \\
\hline \multirow[t]{3}{*}{ Noordzij et al. } & 1991 & 82 & non-invasive, G2 & 73 & bladder & G1-2, pTa-1 \\
\hline & & & & 79,81 & urethra $\times 2$ & G1, non-invasive \\
\hline & & & & 82 & rt. ureter & unknown \\
\hline Ralph et al. & 1991 & 84 & invasive, poorly diff. & 70 & bladder $\times$ several times & G1-2, pT1 \\
\hline \multirow[t]{3}{*}{ Shigeta et al. } & 1994 & 83 & non-invasive, G2 & 80 & 1t. renal pelvis & G2, pT3 \\
\hline & & & & 81 & lt. ureter & G2, pT1b \\
\hline & & & & 81,82 & bladder $\times 5$ & G2 \\
\hline Bass et al. & 1994 & 72 & non-invasive, well diff. & 68,70 & bladder $\times 2$ & G1-2, pTa-1b \\
\hline \multirow[t]{2}{*}{ Jendresen et al. } & 1997 & 61 & non-invasive, G2 & 59 & lt. renal pelvis & G2, pT1b \\
\hline & & 62 & non-invasive, G2 & 59,60 & bladder $\times 2$ & non-invasive, G3 \\
\hline Singer et al. & 1998 & 59 & non-invasive, G1-2 & 55 & bladder $\times 2$ & G1-2>G3, pT1b \\
\hline Bulbul et al. & 1999 & 74 & invasive, G2 & 68 & bladder & invasive, G2 \\
\hline Kato et al. & 1999 & 72 & non-invasive, G2 & 70 & lt. ureter & G2, pT2 \\
\hline Kasai et al. & 2001 & 82 & non-invasive G1-2 & 81,82 & bladder $\times 2$ & G1-2, pT1b \\
\hline Kumar et al. & 2001 & 58 & TCC & 52 & bladder & superficial \\
\hline \multirow[t]{3}{*}{ Okada et al. } & 2004 & 74 & invasive, G3 & 57 & bladder $\times 3$ & G1-2, pTa \\
\hline & & & & 68 & rt. ureter & G3, pT3 \\
\hline & & & & 71 & bladder & G3, pT2 \\
\hline \multirow[t]{3}{*}{ Mondaini et al. } & 2005 & 80 & G2 & 72 & bladder $\times 3$ & $\mathrm{G} 2, \mathrm{pTa}$ \\
\hline & & 82 & invasive, G3 & 77 & lt. ureter & unknown \\
\hline & & & & 79 & bladder & $\mathrm{G} 2, \mathrm{pTa}$ \\
\hline \multirow[t]{4}{*}{ our report } & 2008 & 71 & G3, pT2 & 68 & lt. renal pelvis & G2, pT3 \\
\hline & & & & & bladder & $\mathrm{G} 2, \mathrm{pT} 1$ \\
\hline & & & & & urethra & G2, pT1 \\
\hline & & & & 69,70 & bladder $\times 2$ & G2, pT1 \\
\hline
\end{tabular}

suggested that the mechanism of vaginal metastasis of urothelial cancer might involve seeding due to frequent endoscopic procedures and transfer of urine.

The pathogenesis of vaginal implantation of urothelial carcinoma and lymphatic or vascular spread of tumor cells to the vaginal epithelium is difficult to postulate, even if an urothelial cancer of the upper or lower urinary tract has been curatively treated. Okada et al. $^{7}$ have suggested that G3 vaginal metastasis is due to seeding of invasive G3 urothelial carcinoma, although there was history of G1-2 bladder cancer in the past. Mondaini et al. ${ }^{5}$ have suggested that the G2 urothelial carcinoma seeded in the vagina upgraded in G3. They concluded that a difference in grade had developed between the vaginal tumor and the urinary tract tumor but that the vaginal tumor was due to metastasis of the urothelial carcinoma.

In the present case the tumor recurred repeatedly in the bladder after the cancer of the left renal pelvis was treated, and endoscopic procedures and intravesical instillation were performed repeatedly. A papillary tumor also appeared on the external urethral orifice. The patient had complained of gross hematuria, and urine cytologic examination was positive for malignant cells, although urothelial cancer did not subsequently recur in the upper or lower urinary tract. It was unclear whether the cancer had seeded the vagina when the tumor was removed from the external urethral meatus or whether it had already been transferred to the vagina when the tumor of the external urethral meatus appeared. Circumstances that allowed easy metastatic seeding to the vagina during voiding dribbling may have arisen because the patient's urinary stream changed when the tumor of the external urethral meatus was removed.

We believe that gross hematuria and tumor cells in the urine appeared because the urine mixed with 
atypical genital bleeding from the vaginal tumor. We cannot exclude the possibility of a primary vaginal tumor and lymphatic or vascular transfer of invasive pelvic cancer, but seeding of tumor cells to downstream tissues during urination and resection seemed the only plausible mechanism for the appearance of the vaginal tumor because histological examination showed that the tumor had invaded the muscle layer from the vaginal mucosa. The invasive, high-grade vaginal tumor might have transferred lymphogenously to the left inguinal regional lymph node.

Generally, observation of urothelial cancer involves examination for the presence of recurrent tumors in the upper and lower urinary tract, intrapelvic and paraaortic adenopathy, and bone metastasis, but the vagina is not examined. Our case suggests that vaginal metastasis should be considered if positive urinary cytology is obtained without tumor recurrence, including carcinoma in $s i t u$, in the upper and lower urinary tract.

Acknowledgments: We thank Dr. Hideaki Ishii, Department of Pathology, Nippon Medical School Musashi Kosugi Hospital, for providing expert advice on pathology.

\section{References}

1. Chin JL, Wolf RM, Huben RP, Pontes JE: Vaginal recurrence after cystectomy for bladder cancer. J Urol 1985; 134: 58-61.

2. Ralph DJ, Chapple CR, Evans JW: Seeding of transitional cell carcinoma within the vagina. $\mathrm{Br} \mathrm{J}$ Urol 1991; 67: 660-661.

3. Jendresen MB, Kvist E, Glenthoj A: Papillary transitional cell tumour in the vagina. Scand J Urol Nephrol 1997; 31: 107-108.

4. Kasai T, Moriyama K, Tsuji M, Uema K, Sakurai N: A case of vaginal implantation of transitional cell carcinoma of the bladder. Nippon Hinyokika Gakkai Zasshi 2001; 92: 538-541.

5. Mondaini N, Giubilei G, Raspollini MR, Crisci A, Orlando V: Recurrence of vaginal implantation of transitional cell carcinoma of the urinary tract. Gynecol Oncol 2005; 97: 669-670.

6. Kumar R, Kumar S, Hemal AK: Vaginal and omental metastasis from superficial bladder cancer. Urol Int 2001; 67: 117-118.

7. Okada Y, Nishiyama H, Nakashima M, et al.: A case of vaginal metastasis of transitional cell carcinoma. Hinyokika Kiyo 2004; 50: 283-286.

8. Noordzij JW, Dabhoiwala NF, Dereijke ThM, Tweel JGV: Vulvar and vaginal implantation of transitional cell carcinoma of the urinary tract. Br J Urol 1991; 67: 102-103.

(Received, February 28, 2008)

(Accepted, July 4, 2008) 